Atmos. Chem. Phys., 2, 99-101, 2002

www.atmos-chem-phys.org/acp/2/99/

\title{
Reply to: "Tropical cirrus and water vapor: an effective Earth infrared iris feedback?"
}

\author{
M.-D. Chou ${ }^{1}$, R. S. Lindzen ${ }^{2}$, and A. Y. Hou ${ }^{1}$ \\ ${ }^{1}$ Laboratory for Atmospheres, NASA/Goddard Space Flight Center, Greenbelt, Maryland 20771, USA \\ ${ }^{2}$ Department of Earth, Atmospheric, and Planetary Sciences, Massachusetts Institute of Technology, Cambridge, \\ Massachusetts 02139, USA
}

Received: 21 November 2001 - Published in Atmos. Chem. Phys. Discuss.: 14 February 2002

Revised: 19 April 2002 - Accepted: 19 April 2002 - Published: 30 May 2002

\begin{abstract}
In assessing the iris effect suggested by Lindzen et al. (2001), Fu et al. (2002) found that the response of highlevel clouds to the sea surface temperature had an effect of reducing the climate sensitivity to external radiative forcing, but the effect was not as strong as LCH found. The approach of FBH to specifying longwave emission and cloud albedos appears to be inappropriate, and the derived cloud optical properties may not have real physical meaning. The cloud albedo calculated by FBH is too large for cirrus clouds and too small for boundary layer clouds, which underestimates the iris effect.
\end{abstract}

\section{Introduction}

In assessing the iris effect suggested by Lindzen et al. (2001) (hereafter LCH), Fu et al. (2002) (hereafter FBH) found that the response of high-level clouds to the sea surface temperature had an effect of reducing the climate sensitivity to external radiative forcing, but the effect was not as strong as LCH found. This weaker reduction in climate sensitivity was due to the smaller contrasts in albedos and effective emitting temperatures between cirrus clouds and the neighboring regions. FBH specified the albedos and the outgoing longwave radiation (OLR) in the LCH 3.5-box radiative-convective model by requiring that the model radiation budgets at the top of the atmosphere (TOA) be consistent with that inferred from the Earth Radiation Budget Experiment (ERBE) (Barkstrom, 1984). In point of fact, the constraint by radiation budgets alone is not sufficient for deriving the correct contrast in radiation properties between cirrus clouds and the neighboring

Correspondence to: M.-D. Chou

(chou@climate.gsfc.nasa.gov) regions, and the approach of FBH to specifying those properties is, we feel, inappropriate for assessing the iris effect.

\section{Albedo contrast between cirrus and boundary-layer clouds}

In the LCH 3.5-box model for studying the iris effect, the tropics is divided into a moist region and a dry region. Each region covers half of the tropics. The moist region is further divided into a region covered with high-level cirrus clouds (cloudy moist) and a region without cirrus clouds (clear moist). The areal coverage of the former is assumed to be $22 \%$ of the tropics and the latter $28 \%$. The low-level boundary clouds are assumed to have an areal coverage of $25 \%$ throughout the tropics. In the cloudy-moist region, the low-level boundary clouds overlap with the high-level cirrus clouds. The iris effect depends primarily on the contrast among the longwave emission and albedo of the three tropical regions. The specification of the areal coverage, as well as the specification of the effective emitting temperatures and cloud albedos, are required to be consistent with the overall ERBE radiation budgets.

FBH estimated the OLR of the dry region and the clearmoist region from radiation model calculations with appropriate temperature and humidity profiles for the tropics. The OLR of the cloudy-moist region was then derived by requiring that the mean OLR of the model tropics be the same as that of the ERBE, which is $255 \mathrm{~W} \mathrm{~m}^{-2}$. Based on both ERBE data and model calculations, they assumed that the effect of high cirrus clouds on the shortwave and longwave radiation (or cloud radiative forcing, CRF) nearly cancelled each other, and the net effect on the TOA radiation budget was negligible. They then derived the albedos of the highlevel cirrus clouds and the low-level boundary-layer clouds 
by requiring that the following two conditions were met. (1) The radiative forcing of high-level clouds for shortwave radiation and longwave radiation was equal in the cloudy-moist region. (2) The mean albedo of the tropics was equal to the ERBE-inferred value of 0.241 .

Cirrus clouds reduce both the longwave cooling and shortwave heating of the Earth. The magnitude of these two competing effects depends on the optical thickness of the clouds. Thin cirrus with an optical thickness of, say $<1$, in the visible spectral region are relatively transparent to shortwave radiation but not necessarily transparent to the longwave radiation. Thus, thin cirrus clouds have a stronger longwave warming effect than a shortwave cooling effect, which leads to a net warming effect on the climate. On the other hand, thick cirrus clouds are highly reflective to shortwave radiation and generally have a net cooling effect on the climate. The overall effect of cirrus on the Earth radiation budget depends strongly on the areal coverage of thin cirrus clouds relative to that of thick cirrus clouds.

There are two sources of thin cirrus clouds. One is the detrainment of deep convective anvil clouds which spread, precipitate, and evaporate to become thin cirrus in the neighborhood of cumulus cloud clusters. The other is the thick cumulus clouds that are left behind propagating large-scale atmospheric disturbances and decay rapidly to become thin cirrus and contribute to the supply of water vapor in the upper troposphere. The upper tropospheric water vapor may later form thin cirrus clouds due to atmospheric wave motions (Boehm and Verlinde, 2000). These thin cirrus clouds are widespread and can persist for a long period of time due to large-scale lifting of air in the tropics (Boehm et al., 1999). Although it is generally believed that thin cirrus are widespread in the tropics, detection and retrieval of these clouds using satellite-measured radiances are largely unreliable, as it is very difficult to differentiate thin cirrus clouds from broken clouds at lower levels. As a result, the net effect of high-level cirrus clouds on the earth radiation budget is hard to assess, either directly from satellite radiation measurements, or coupled with radiation model calculations as was done by FBH. The assertion by FBH that the radiative forcing of high clouds in the shortwave and longwave spectral regions cancelled each other has yet to be actually validated with reliable cloud and radiation data.

The model climate sensitivity, as calculated by LCH, depends only weakly on the subjectively specified areal extent of the three tropical regions. However, if the longwave emission of the three regions and the shortwave albedos of clouds are derived by imposing certain radiation budget constraints as in $\mathrm{FBH}$, these parameters will be sensitive to the subjectively specified areal coverage of the three regions, and so is the model climate sensitivity. Figure 1 shows the longwave emission of the cloudy-moist region as a function of the cirrus cloud fraction relative to the entire tropics. It is derived by assuming that OLR in the dry and clear-moist regions are, respectively, $293 \mathrm{~W} \mathrm{~m}^{-2}$ and $268 \mathrm{~W} \mathrm{~m}^{-2}$ as calculated

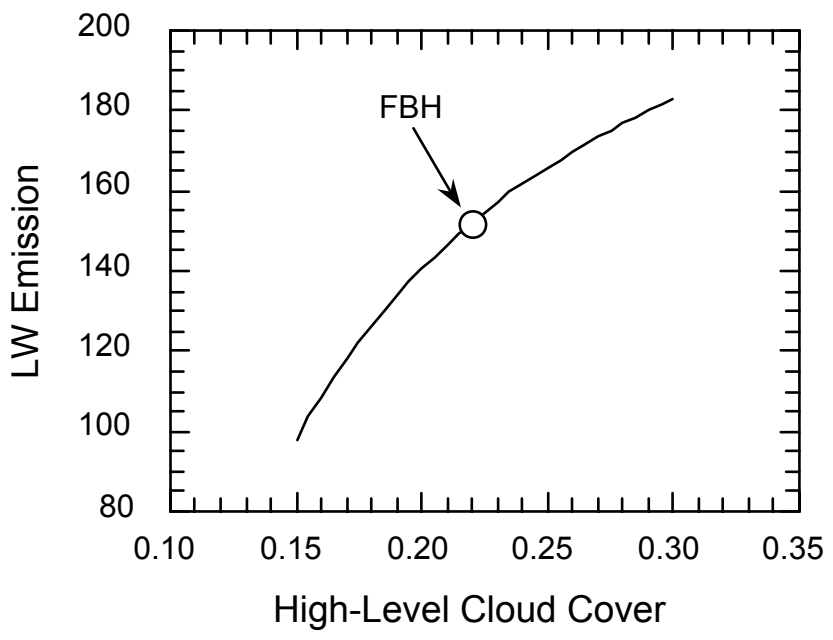

Fig. 1. Longwave emission $\left(\mathrm{W} \mathrm{m}^{-2}\right)$ of the cloudy-moist region as a function of the fractional high-level cloud cover relative to the entire tropics. The circle represents the OLR and the high-level cloud cover derived by FBH. See the text for details.

by $\mathrm{FBH}$, and an areal coverage of $50 \%$ of the dry region as assumed by LCH. To match the mean OLR of $255 \mathrm{~W} \mathrm{~m}^{-2}$ in the tropics inferred by ERBE, FBH calculated the OLR to be $154 \mathrm{~W} \mathrm{~m}^{-2}$ for a $22 \%$ coverage of the cloudy-moist region, which is marked by a circle in the figure. If the cloudy-moist region increases by $5 \%$ from $22 \%$ to $27 \%$, the longwave emission would increase by $22 \mathrm{~W} \mathrm{~m}^{-2}$ according to Figure 1. Considering the large extent of thin cirrus clouds, the highlevel cloud cover of $27 \%$ in the tropics is not at all unrealistic. The albedos of both high clouds and low clouds will have to change if the FBH approach is to be followed, which requires that the net CRF of high clouds be zero and the mean albedo of the tropical region be equal to the ERBE-inferred value of 0.241 . The contrast between the cloud albedos will vary with the subjectively specified coverage of the three regions, and the simulated climate sensitivity may not have any physical meaning.

Both the contrast among the longwave emission of the three tropical regions and the contrast between the albedos of the high-level cirrus and low-level boundary layer clouds derived by FBH are smaller than that specified by $\mathrm{LCH}$. LCH assigned an albedo of 0.24 for high cirrus clouds, and a significantly higher albedo of 0.42 was assigned for low boundary clouds. In contrtast, FBH derived a nearly constant albedo for high-level and low-level clouds 0.342 for the former and 0.331 for the latter. FBH found that these differences in cloud albedos and differences in longwave emission caused the negative feedback factor as estimated by $\mathrm{LCH}$ to decrease by $50 \%$.

The optical thickness of low-level boundary clouds varies with fractional cloud cover. The visible optical thickness inferred from the high spatial-resolution Landsat imagery of marine boundary layer clouds ranges from $\sim 5$ for scattered 
cumulus to $\sim 20$ for overcast stratocumulus (Barker et al., 1996). Szczodrak et al. (2001) derived the optical thickness and the effective radius of marine stratocumulus clouds using the NOAA Advanced Very High Resolution Radiometer (AVHRR) radiance measurements over the eastern Pacific Ocean and the Southern Ocean near Tasmania. They found that the majority of clouds have a visible optical thickness in the range 5-30. Our model calculations show that, for a solar zenith angle of $60^{\circ}$, the albedo corresponding to these optical thickness ranges from 0.35 to 0.60. Buriez et al. (2001) studied the cloud optical thickness retrieved from the Advanced Earth Orbiting Satellite-Polarization and Directionality of the Earth's Reflectances (ADEOS-POLDER) observations. They found that overcast low-level clouds were bright with an albedo of $0.4-0.7$. Thus, the albedo of 0.42 used by $\mathrm{LCH}$ is in agreement with these satellite observations. The specification of the albedo of 0.24 by LCH for high clouds takes into consideration of the extended coverage of thin cirrus clouds in the tropics, which have a low albedo. On the other hand, the albedos of 0.342 for high clouds and 0.331 for low clouds as used by FBH are inconsistent with observations.

LCH specified the OLR to be $263 \mathrm{~W} \mathrm{~m}^{-2}$ in the clearmoist region and $303 \mathrm{~W} \mathrm{~m}^{-2}$ in the dry region. Whereas FBH calculated the OLR and albedo of these regions to be $268 \mathrm{~W} \mathrm{~m}^{-2}$ and $293 \mathrm{~W} \mathrm{~m}^{-2}$, respectively, using a radiation model. When we apply the OLR values computed by FBH to the LCH 3.5-box model, the negative feedback factor is reduced only moderately by $\sim 20 \%, 10 \%$, and $16 \%$ for $\gamma=$ $1.0,0.5$, and 0 , respectively. In commenting on this response, Baker (2002) suggests that the contrast of the TOA radiation between the tropical moist-cloudy and dry regions is highly overestimated in LCH. From the ERBE data, she finds that for a given latitude band, the longitudinal contrast of the net TOA radiation does not exceed $40 \mathrm{~W} \mathrm{~m}^{-2}$. This number is significantly smaller than the radiation contrast of $110 \mathrm{~W} \mathrm{~m}^{-2}$ between the moist-cloudy and dry regions in LCH. Baker then concludes that the feedback factor of climate sensitivity as estimated by LCH is significantly exaggerated.

In the ERBE data archive, the TOA radiation budgets are monthly mean values averaged over $2.5^{\circ} \times 2.5^{\circ}$ latitudelongitude boxes. Tropical cloud systems associated with easterly waves and Madden-Julian Oscillations propagate in zonal directions. The MJO's, for example, propagate from west to east at a speed of $\sim 5-6 \mathrm{~m} \mathrm{~s}^{-1}$. With this speed, cloud systems propagate by $>250 \mathrm{~km}$ within a day. Therefore, one can expect that the possibility of a $2.5^{\circ} \times 2.5^{\circ}$ latitude-longitude box in the convection region to be continuously and totally covered by clouds in a one-month period is nearly 0 . The ERBE monthly radiation in convective regions represents a mixture of radiation of both the moistcloudy and moist-clear regions, and the net TOA radiation of the cloudy-moist region cannot be identified from the longitudinal distributions of the ERBE fluxes, as is attempted by
Baker (2002).

\section{Conclusions}

The approach of FBH to specifying longwave emission and cloud albedos appears to be inappropriate for studying the iris effect. These radiation properties are sensitive to the subjectively specified areal coverage of the three tropical regions, and the derived properties by FBH may not have real physical meaning. From the point of view that thin cirrus are widespread in the tropics and that low boundary clouds are optically thick, the cloud albedo calculated by FBH is too large for cirrus clouds and too small for boundary layer clouds. The near-zero contrast in cloud albedos derived by FBH has the effect of underestimating the iris effect. On the other hand, the contrast of longwave emission among the three regions as derived by FBH is smaller than that of $\mathrm{LCH}$. If the longwave emission derived by FBH is appropriate, then LCH may indeed have overestimated the iris effect somewhat, though hardly by as much as that suggested by FBH.

Acknowledgements. The work of M.-D. Chou was supported by the Radiation Science Program, NASA/Office of Earth Science. The efforts of R. S. Lindzen were supported by Grant DE-FG0201ER63257 from the Department of Energy.

\section{References}

Baker, M.: Interactive comment on "Reply to: 'Tropical cirrus and water vapor: an effective Earth infrared iris feedback?'”, Atmos. Chem. Phys. Discuss., 2, S59-S62, 2002.

Barker, W. H., Wielicki, B. A., and Parker, L.: A parameterization for computing gridaveraged solar fluxes for inhomogeneous marine boundary layer clouds. Part II: Validation using satellite data, J. Atmos. Sci., 53, 2304-2316, 1996.

Barkstrom, B. R.: The Earth Radiation Budget Experiment (ERBE), Bull. Amer. Meteorol. Soc., 65, 1170-1185, 1984.

Boehm, M. and Verlinde, J.: Stratosphereic influence on upper tropospheric tropical cirrus; Geophy. Res. Let., 27, 3209-3212, 2000.

Boehm, M., Verlinde, J., and Ackerman, T. P.: On the maintenance of high tropical cirrus, J. Geophy. Res., 104, 24 423-24433, 1999.

Buriez, J.-C., Doutriaux-Boucher, M., and Parol, F.: Angular variability of the liquid water cloud optical thickness retrieved from ADEOS-POLDER, J. Atmos. Sc., 58, 3007-3018, 2001.

Fu, Q., Baker, M., and Hartman, D. L.: Tropical cirrus and water vapor: an effective Earth infrared iris feedback? Atmos. Chem. Phys., 2, 31-37, 2002.

Lindzen, R. S., Chou, M.-D., and Hou, A. Y.: Does the earth have an adaptive infrared iris? Bull. Amer. Met. Soc., 82, 417-432, 2001.

Szczodrak, M., Austin, P. H., and Krummel, P. B.: Variability of optical depth and effective radius in marine stratocumulus clouds, J. Atmos. Sci., 58, 2912-2926, 2001. 